\title{
Quaternionic Formalism for Non-Abelian Gauge Theory of Dyons and Gravito-Dyons
}

\author{
Gaurav Karnatak, P.S. Bisht and O.P.S. Negi \\ Department of Physics, K.U. S.S.J. Campus, Almora, Uttarakhand \\ Email: gauravkarnatak2009@yahoo.in
}

\begin{abstract}
Starting with the Quaternion formulation of $S U(2) \times U(1)$ gauge theory of dyons and gravitodyons, it is shown that the formulation characterizes the abelian and non-Abelian gauge structure of dyons and gravito-dyons in terms of purely real and imaginary units of quaternion.
\end{abstract}

Keywords: Quaternions, Dyons, Non-abelian gauge theories.

\section{Introduction}

The unified electromagnetism and gravitation interms of single parameter via quaternion gauge formalism in a unique and consistent manner. The isomorphic $S U(2) \times U(1)$ gauge structure of general linear quaternion gauge group of dyons and gravito-dyons is different from the well known gauge group structure of Salam-Weinberg theory of electroweak interactions. Octonions are widely used for the understanding of unification structure of successful gauge theory of fundamental interaction. Recently, the quaternionic formulation of Yang-Mill's field equations and Octonion reformulation of quantum chromodynamics (QCD) by taking magnetic monopoles and dyons (particles carrying electric and magnetic charges) into account. In this Chapter, we have developed the quaternion formulation of $S U(2) \times U(1)$ gauge theory of dyons and gravito-dyons.

\section{Quaternion formulation of $S U(2) \times U(1)$ gauge theory of dyons and gravito-dyons}

The two gauge fields $\left\{\mathrm{A}_{\mu}\right\}$ and $\left\{\mathrm{B}_{\mu}\right\}$ respectively associated with electric charge
$U(1)_{e}$ and magnetic monopole $U(1)_{g} .\left\{\mathrm{M}_{\mu}\right\}$ and $\left\{\mathrm{N}_{\mu}\right\}$ associated with gravitational charge $U(1)_{m}$ and Heavisidian monopole $U(1)_{h}$. So, the generalized potential of dyons in $U(1) \times U(1)$ gauge theory be written as $\left\{V_{\mu}\right\}=\left[\begin{array}{cc}\mathrm{A}_{\mu} & 0 \\ 0 & \mathrm{~B}_{\mu}\end{array}\right]$

$\left\{\mathrm{Z}_{\mu}\right\}=\left[\begin{array}{cc}\mathrm{M}_{\mu} & 0 \\ 0 & \mathrm{~N}_{\mu}\end{array}\right]$

If $\mathfrak{I}$ is a quaternion, the quaternion Qderivative acting on a quaternion $\mathfrak{I}$ is described as

$\mathfrak{I}_{I I, \mu}=\mathfrak{I}_{, \mu}+\left\lfloor\Gamma_{\mu}, \mathfrak{I}\right\rfloor$

Where $\mathfrak{I}_{\mu}$ is partial derivative and $\Gamma_{\mu}$ is defined as,

$\Gamma_{\mu}=i \mathrm{Y}_{\mu} \cdot \tau=-\mathrm{Y}_{\mu} \cdot e=\sum_{j=1}^{3} \mathrm{Y}_{\mu}^{j} e_{j}$

The gauge potential and gauge field strength for electric charge and dual charge (i.e. 
monopole) in quaternionic version of $U(1) \times S U(2)$ gauge theory as

$$
\begin{aligned}
& \left.\mathrm{A}_{\mu}=\mathrm{A}_{\mu}^{0} e^{0}+\mathrm{A}_{\mu}^{j} e^{j}=\mathrm{A}_{\mu}^{0} \hat{1}+\frac{1}{i} \mathrm{~A}_{\mu}^{j} \tau_{j}\right) \\
& (\forall \mathrm{j}=1,2,3) \\
& F_{\mu \nu}=F_{\mu \nu}^{0} e^{0}+F_{\mu \nu}^{j} e^{j}=F_{\mu \nu} \hat{1}+\frac{1}{i} F_{\mu \nu}^{j} \tau_{j} \\
& \mathrm{~B}_{\mu}=\mathrm{B}_{\mu}^{0} e^{0}+\mathrm{B}_{\mu}^{j} e^{j}=\mathrm{B}_{\mu}^{0} \hat{1}+\frac{1}{i} \mathrm{~B}_{\mu}^{j} \tau_{j} \\
& \forall \mathrm{j}=1,2,3) \\
& \tilde{F}_{\mu \nu}=\tilde{F}_{\mu \nu}^{0} e^{0}+\tilde{F}_{\mu \nu}^{j} e^{j}=\tilde{F}_{\mu \nu} \hat{1}+\frac{1}{i} \tilde{F}_{\mu \nu}^{j} \tau_{j}
\end{aligned}
$$

Similarly the gauge potential and gauge field strength for gravitational charge in quaternionic version of $U(1) \times S U(2)$ gauge theory as

$$
\mathrm{M}_{\mu}=\mathrm{M}_{\mu}^{0} e^{0}+\mathrm{M}_{\mu}^{j} e^{j}=\mathrm{M}_{\mu}^{0} \hat{1}+\frac{1}{i} \mathrm{M}_{\mu}^{j} \tau_{j}
$$

$$
(\forall \mathrm{j}=1,2,3)
$$$$
f_{\mu \nu}=f_{\mu \nu}^{0} e^{0}+f_{\mu \nu}^{j} e^{j}=f_{\mu \nu} \hat{1}+\frac{1}{i} f_{\mu \nu}^{j} \tau_{j}
$$$$
\mathrm{N}_{\mu}=\mathrm{N}_{\mu}^{0} e^{0}+\mathrm{N}_{\mu}^{j} e^{j}=\mathrm{N}_{\mu}^{0} \hat{1}+\frac{1}{i} \mathrm{~N}_{\mu}^{j} \tau_{j}
$$

$$
\begin{aligned}
& (\forall \mathrm{j}=1,2,3) \\
& \tilde{f}_{\mu \nu}=\tilde{f}_{\mu \nu}^{0} e^{0}+\tilde{f}_{\mu \nu}^{j} e^{j}=\tilde{f}_{\mu \nu} \hat{1}+\frac{1}{i} \tilde{f}_{\mu \nu}^{j} \tau_{j}
\end{aligned}
$$

Where $e_{j} \Leftrightarrow-i \tau_{j}$ and $\tau_{j}$ usual Pauli-spin matrices. Hence for the $U(1) \times S U(2)$ quaternion gauge theory, the gauge potential and gauge field strength be expressed as

$$
\begin{gathered}
\mathrm{K}_{\mu}=\left(\begin{array}{cc}
\mathrm{A}_{\mu} e^{0}-i \sum_{a=1}^{3} \mathrm{~A}_{\mu}^{a} \tau^{a} & 0 \\
0 & \mathrm{~B}_{\mu} e^{0}-i \sum_{a=1}^{3} \mathrm{~B}_{\mu}^{a} \tau^{a}
\end{array}\right) \\
\mathrm{X}_{\mu \nu}=\left(\begin{array}{cc}
-i e\left(F_{\mu \nu}-i \sum_{a=1}^{3} F_{\mu}^{a} \tau^{a}\right) & 0 \\
0 & -i g\left(\widetilde{F}_{\mu \nu}-i \sum_{a=1}^{3} \widetilde{F}_{\mu \nu}^{a} \tau^{a}\right)
\end{array}\right)(1+13) \\
=-i\left(\begin{array}{cc}
e F_{\mu \nu} & 0 \\
0 & g \widetilde{F}_{\mu \nu}
\end{array}\right)
\end{gathered}
$$

Similarly, the $U(1) \times S U(2)$ quaternion gauge theory, the gauge potential and gauge field strength of gravito-dyons may be expressed as

$$
\begin{aligned}
& L_{\mu}=\left(\begin{array}{cc}
\mathbf{M}_{\mu} e^{0}-i \sum_{a=1}^{3} \mathbf{M}_{\mu}^{a} \tau^{a} & 0 \\
0 & \mathbf{N}_{\mu} e^{0}-i \sum_{a=1}^{3} \mathbf{N}_{\mu}^{a} \tau^{a}
\end{array}\right) \\
& Y_{\mu \nu}=\left(\begin{array}{cc}
-i m\left(f_{\mu \nu}-i \sum_{a=1}^{3} f_{\mu \nu}^{a} \tau^{a}\right) & 0 \\
0 & -i h\left(\tilde{f}_{\mu \nu}-i \sum_{a=1}^{3} \tilde{f}_{\mu \nu}^{a} \tau^{a}\right)
\end{array}\right) \\
& =-i\left(\begin{array}{cc}
e f_{\mu \nu} & 0 \\
0 & g \tilde{f}_{\mu \nu}
\end{array}\right)
\end{aligned}
$$

The commutation relation of covariant derivative for $U(1) \times S U(2)$ gauge theory of dyons and gravito-dyons be given as

$$
\begin{aligned}
& {\left[D_{\mu}, D_{v}\right]=\left[\begin{array}{cc}
\left(F_{\mu \nu}-i \sum_{a=1}^{3} F_{\mu \nu}^{a} \tau^{a}\right) & 0 \\
0 & \left(\tilde{F}_{\mu \nu}-i \sum_{a=1}^{3} \tilde{F}_{\mu \nu}^{a} \tau^{a}\right)
\end{array}\right]=X_{\mu \nu}} \\
& {\left[\Delta_{\mu}, \Delta_{\nu}\right]=\left[\begin{array}{cc}
\left(f_{\mu \nu}-i \sum_{a=1}^{3} f_{\mu \nu}^{a} \tau^{a}\right) & 0 \\
0 & \left(\tilde{f}_{\mu \nu}-i \sum_{a=1}^{3} \tilde{f}_{\mu \nu}^{a} \tau^{a}\right)
\end{array}\right]=Y_{\mu \nu}}
\end{aligned}
$$

Which yields the correct field equation for non-Abelian gauge theory of dyons and gravito-dyons and give rise to its extended structure, which reproduces 


$$
\begin{aligned}
& D_{\nu} X_{\mu \nu}=\left[\begin{array}{cc}
e j_{\mu} & 0 \\
0 & g k_{\mu}
\end{array}\right]=J_{\mu} \\
& J_{\mu}=\left(\begin{array}{cc}
e\left(j_{\mu} e^{0}-i \sum_{a=1}^{3} j_{\mu}^{a} \tau^{a}\right) & 0 \\
0 & g\left(k_{\mu} e^{0}-i \sum_{a=1}^{3} k_{\mu}^{a} \tau^{a}\right)
\end{array}\right) \\
& D_{\nu} Y_{\mu \nu}=\left[\begin{array}{cc}
e l_{\mu} & 0 \\
0 & g m_{\mu}
\end{array}\right]=-K_{\mu} \\
& K_{\mu}=\left(\begin{array}{c}
m\left(l_{\mu} e^{0}-i \sum_{a=1}^{3} l_{\mu}^{a} \tau^{a}\right) \\
0 \\
h\left(n_{\mu} e^{0}-i \sum_{a=1}^{3} n_{\mu}^{a} \tau^{a}\right.
\end{array}\right)
\end{aligned}
$$

Thus the generalized Q-four current of dyons in quaternion $U(1) \times S U(2)$ gauge formalism leads to the abelian and non-Abelian nature current and leads to the Noetherian conservation of current of dyons and gravitodyons as

$$
J_{\mu I I \mu}=0 \quad \text { and } \quad K_{\mu I I \mu}=0
$$

Which shows the resemblance with Noetherian current analogous to the continuity equation in abelian gauge theory of dyons and gravito-dyons. In general this current is no more conserved in terms of its ordinary derivative to Q-derivative the generalized quaternionic current of dyons and gravito dyons becomes conserved.

\section{Discussion}

The non-Abelian gauge theory of dyons for weak and strong interactions has been developed consistently in terms of two gauge potentials namely electric and magnetic ones. Starting with the quaternion formulation of $U(1) \times S U(2)$ gauge theory of dyons and gravito-dyons. It is shown that the formulation characterizes the abelian and non-Abelian structure of dyons and gravito-dyons in terms of pure real and imaginary unit quaternion.

\section{References}

Bisht, P. S., Negi, O. P. S., Rajput, B. S., 1990, Ind. J. Pure and Appl. Phys., 28, 157. Bisht, P. S., Negi, O. P. S., Rajput, B. S., 1993, Ind. J. Pure and Appl. Phys., 24, 543. Ross, D. K., 1983, J. Phys. Math. Gen, A16, 1331.

Bisht, P. S., Negi, O. P. S., Rajput, B. S., 1991, Prog.Theo.Phys. 85, 157.

Rajput, B. S., 1982, Lett. Nuovo Cimento, 35, 205.

Rajput, B. S., 1984, J. Math. Phys., 25, 351.

Karnatak, Gaurav., Bisht, P. S., Negi., O. P. S. 2014, Int. J. of Mod. App Phys, 4(2), 83. 